Pacific Journal of Mathematic 


\title{
CRAWLEY'S COMPLETION OF A CONDITIONALLY UPPER CONTINUOUS LATTICE
}

\author{
WILliam H. CORNISH
}

\begin{abstract}
Crawley's completion (the lattice of all complete ideals) of a conditionally upper continuous lattice $L$ is an upper regular homomorphic image of the lattice of ideals of $L$. After examining the consequences of this result, Crawley's completion is characterized both as a completion of $L$ and as the minimal upper continuous extension of $L$ with respect to upper regular homomorphisms.
\end{abstract}

1. Introduction. A lattice is conditionally upper continuous if for any $x \in L$ and any (upward) directed subset $\left\{x_{\alpha}\right\}$ such that $V x_{\alpha}$ exists then $\mathrm{V} x \wedge x_{\alpha}$ exists and $x \wedge \mathrm{V} x_{\alpha}=\mathrm{V} x \wedge x_{\alpha}$. An upper continuous lattice is a complete conditionally upper continuous lattice.

The empty subset $\varnothing$ will be regarded as an ideal of lattice $L$ if and only if $L$ possesses no smallest element. Thus the lattice $J(L)$ of ideals of $L$ is a complete lattice whose smallest element is either $\varnothing$ or $\{0\}$ according to whether $L$ is unbounded below or $L$ has a smallest element 0. A complete ideal is an ideal which is closed under the formation of existing joins of its elements. The set $K(L)$ of all complete ideals is a closure system on $L$ and is thus a complete lattice which possesses the same smallest and largest elements as $J(L)$.

A function between two partially ordered sets is called upper regular if it preserves existing joins. Lower regular functions are defined dually while a function which is both upper and lower regular is simply called regular. The natural function $\kappa: L \rightarrow K(L)$, defined by $\kappa(x)=\{y \in L: y \leqq x\}$, is always regular.

In [2] Crawley proved that $K(L)$ is upper continuous if and only if $L$ is conditionally upper continuous, together with the most interesting fact that $L$ and $K(L)$ satisfy precisely the same identities when $L$ is conditionally upper continuous. We first show that Crawley's results are direct consequences of the fact that if $L$ is conditionally upper continuous then $K(L)$ is an upper regular lattice-homomorphic image of $J(L)$. This last fact leads to a characterization of upper continuous lattices as a type of retract of algebraic lattices (complete compactly generated lattices), a natural proof of J. Schmidt's theorem [13] that a conditionally upper continuous distributive lattice is infinitely distributive, and a simple way of obtaining an upper regular embedding of an infinitely distributive lattice into a complete Boolean algebra. Here a lattice $L$ is called infinitely distributive if for any $x$ in $L$ and any subset $\left\{x_{\alpha}\right\}$ such that $V x_{\alpha}$ exists then $\mathrm{V} x \wedge x_{\alpha}$ exists 
and $x \wedge \mathrm{V} x_{\alpha}=\mathrm{V} x \wedge x_{\alpha}$; there is no uniform terminology for this concept and so we use the reasonably descriptive term due to Dilworth and McLaughlin [3].

Subsequent to [2], $K(L)$ does not seem to have been studied by any author other than Janowitz [8], [9]. Hence we characterize $K(L)$ and in the last section of this paper examine the case when $K(L)$ is a Boolean algebra.

2. $K(L)$ as a homomorphic image. Let $\langle A\rangle$ denote the complete ideal generated by an ideal $A$ in lattice $L ;\langle A\rangle=\bigcap\{B \in K(L)$ : $A \subseteq B\}$. The meet of an arbitrary set $\left\{J_{\alpha}\right\}$ of complete ideals is their set-theoretic intersection while their join is easily seen to be given by $\mathrm{V}_{K} J_{\alpha}=\left\langle\mathrm{V}_{J} J_{\alpha}\right\rangle$, where $\mathrm{V}_{J}$ denotes the join in $J(L)$. For $x \in L$, the smallest complete ideal containing $x$ is $(x]=\{y \in L: y \leqq x\}$.

Lemma 2.1. For an ideal $A$ in a conditionally upper continuous lattice $L,\langle A\rangle=\left\{x \in L: x=\bigvee A_{1}\right.$ for some ideal $\left.A_{1} \subseteq A\right\}$.

Proof. Let $X=\left\{x \in L: x=\bigvee A_{1}\right.$ for some ideal $\left.A_{1} \subseteq A\right\}$. Suppose $y \in L$ and $y \leqq x$ for some $x \in X$. For a suitable ideal $A_{1} \leqq A, x=$ $\mathrm{V} A_{1}$. As $A_{1}$ is directed and $L$ is conditionally upper continuous $y=$ $y \wedge x=\bigvee y \wedge A_{1}=\mathrm{V}\left((y] \cap A_{1}\right)$. Thus $y \in X$. Now suppose that $S \subseteq$ $X$ and that $z=\mathrm{V} S$ exists. For each $s \in S$ there is an ideal $A_{s} \subseteq A$ such that $s=\mathrm{V} A_{s}$. Hence $z=\mathrm{V}\left(\mathrm{\bigcup}_{s \in S} A_{s}\right)=\mathrm{V}\left(\mathrm{V}_{s \in S} A_{s}\right)$ and so $z \in X$.

Thus $X$ is a complete ideal containing $A$ and it follows that $X=$ $\langle A\rangle$.

We now come to the fundamental characterizations of a conditionally upper continuous lattice. The equivalence of conditions (a), (d), and (e) of the following theorem is known from [2] and [9, Lemma 3.2] yet it is added for the sake of completeness.

THEOREM 2.2. For a lattice $L$ the following are equivalent.

(a) $L$ is conditionally upper continuous.

(b) For any ideals $A$ and $B,\langle A \cap B\rangle=\langle A\rangle \cap\langle B\rangle$.

(c) The function $A \nrightarrow\langle A\rangle$ is an upper regular lattice-homomorphism of $J(L)$ onto $K(L)$.

(d) $K(L)$ is upper continuous.

(e) There exists an upper regular lattice-embedding of $L$ into an upper continuous lattice.

Proof. (a) $\Rightarrow$ (b) Suppose (a) holds and $x \in\langle A\rangle \cap\langle B\rangle$. By 2.1 there are ideals $A_{1} \subseteq A, B_{1} \subseteq B$ such that $x=\mathrm{V} A_{1}=\mathrm{V} B_{1}$. Clearly $x$ is an upper bound of $A_{1} \cap B_{1}$. Let $y$ be another upper bound. 
For any $a \in A_{1}$ and $b \in B_{1}, y \geqq a \wedge b$. Hence $y \geqq \bigvee\left\{a \wedge b: b \in B_{1}\right\}=$ $a \wedge \bigvee B_{1}=a \wedge x$ and it follows that $y \geqq x=x \wedge x=\mathrm{V} A_{1} \wedge x$. Thus $x=\mathrm{V} A_{1} \cap B_{1} \in\langle A \cap B\rangle$. It is now clear that (b) holds.

(b) $\Rightarrow$ (c) Clearly $\left\langle\mathrm{V}_{J} A_{\alpha}\right\rangle \supseteqq \mathrm{V}_{K}\left\langle A_{\alpha}\right\rangle$ for any collection $\left\{A_{\alpha}\right\} \cong J(L)$. On the other hand, $\mathrm{V}_{J} A_{\alpha} \subseteq \mathrm{V}_{J}\left\langle A_{\alpha}\right\rangle$ so that $\left\langle\mathrm{V}_{J} A_{\alpha}\right\rangle \subseteq\left\langle\mathrm{V}_{J}\left\langle A_{\alpha}\right\rangle\right\rangle=$ $\mathrm{V}_{K}\left\langle A_{\alpha}\right\rangle$. In other words the function $A \nrightarrow\langle A\rangle$ is always upper regular and so (c) follows immediately from (b).

$(c) \Rightarrow(d)$ is an immediate consequence of the fact that $J(L)$ is upper continuous.

$(d) \Rightarrow(e)$ is trivial, while $(e) \Rightarrow$ (a) has already been shown in [9, Lemma 3.2].

COROLlary 2.2.1. (Crawley). Let $L$ be a conditionally upper continuous lattice. Then,

(a) for any variety of of lattices, $L \in \mathscr{Y}$ if and only if $K(L) \in \mathscr{Y}$, and

(b) if $p(,, \cdots$,$) is an n-ary lattice polynomial whose evalua-$ tions on $J(L)$ and $K(L)$ are denoted by $p(,, \cdots,)_{J}$ and $p(,, \cdots,)_{K}$ respectively then $p\left(A_{1}, A_{2}, \cdots, A_{n}\right)_{K}=\{x \in L: x=\bigvee B$ for some ideal $\left.B \subseteq p\left(A_{1}, A_{2}, \cdots, A_{n}\right)_{J}\right\}$ for any $n$ complete ideals $A_{1}, A_{2}, \cdots, A_{n}$.

Proof. Both assertions are immediate consequences of 2.2 (c) and Lemma 8 of [6, p. 34].

CoRollary 2.2.2. A lattice $L$ is upper continuous if and only if there exists an algebraic lattice $M$, a lattice-homomorphism $\psi: L \rightarrow$ $M$ and an upper regular lattice-homomorphism $\varphi: M \rightarrow L$ such that $\varphi_{0} \psi$ is the identity function on $L$.

Proof. Suppose $L$ is upper continuous. Take $M=J(L)$ and $\psi$ as given by $\psi(x)=(x]$ for each $x \in L$. As $L$ is upper continuous the canonical embedding $\kappa$ of $L$ into $K(L)$ (recall that $\kappa(x)=(x]$ for each $x \in L)$ is an isomorphism whose inverse $\kappa^{-1}$ is given by $\kappa^{-1}(A)=\mathrm{V} A$ for each $A \in K(L)$. If $\varphi$ is the composition of the function $A \nrightarrow\langle A\rangle$ of 2.2 followed by $\kappa^{-1}$ then the triple $(\psi, M, \varphi)$ has the required properties.

The converse holds since a retract of a complete lattice is complete, an algebraic lattice is upper continuous [1, Lemma 2, p. 187] and the properties of the maps $\varphi$ and $\psi$ then force (conditional) upper continuity.

Along with Smith [14] we shall call a lattice $L$ implicative if for any $a, b \in L$ there exists a necessarily unique element, denoted by $a \rightarrow b$, with the property that $c \wedge a \leqq b$ if and only if $c \leqq a \rightarrow b$ for any $c \in L$. 
CoROLlary 2.2.2. For a lattice $L$ the following are equivalent.

(a) $L$ is distributive and conditionally upper continuous.

(b) $K(L)$ is infinitely distributive.

(c) $L$ is infinitely distributive.

(d) $L$ possesses an upper regular lattice-embedding into a complete Boolean algebra.

(e) $L$ possesses an upper regular lattice-embedding into an implicative lattice.

Proof. (a) $\Rightarrow$ (b) follows from 2.2 since $J(L)$ is infinitely distributive for any distributive lattice $L$. The implications $(b) \Rightarrow(c)$ and (c) $\Rightarrow$ (a) are trivial.

(b) $\Rightarrow$ (d) Let $B(K(L))$ denote the Boolean algebra generated by $K(L)$. Because of (b) the natural map of $K(L)$ into $B(K(L))$ is upper regular; this is proved in exactly the same way as Lemma 13 is established in [6, p. 108]. The embedding of $B(K(L))$ into $A(B(K(L)))$, its complete Boolean algebra of annihilator ideals, is upper regular, c.f. [6, Lemma 12, p. 107], and (d) follows.

$(d) \Rightarrow$ (c) holds since any Boolean algebra is implicative and

$(\mathrm{e}) \Rightarrow$ (c) is easy to prove and its proof will be omitted.

REMARKS. (a) The equivalence of (a) and (c) of 2.2.2 was first established for complete lattices by Schmidt [13, Korollar 7]. His proof is obtained by examining variations of an axiom of Baer. It might be of interest to note that Schmidt's conditions can be made "conditional" and so obtain other characterizations of conditional upper continuity and an alternative proof of (a) $\Leftrightarrow(c)$.

(b) The easy proof of (c) $\Rightarrow$ (d) of 2.2.2 should be compared with complicated proof of the theorem that a lattice which is both infinitely distributive and dual infinitely distributive possesses a regular embedding into a complete Boolean algebra as given by Funayama [4]. Our proof is based on Grätzer's easy proof of Funayama's theorem for the case of a complete lattice. Incidentally we shall see later that the Boolean algebra of annihilator ideals of a Boolean algebra is the Crawley completion; thus we have a very nice description for the Boolean algebra required in (d).

3. Characterizations of $K(L)$. Recall that a subset $S$ of a partially ordered set $P$ is join-dense (meet-dense) in $P$ if and only if each element of $P$ is the join (meet) of its predecessors (successors) in $S$.

THeOREM 3.1. Let $L$ be a conditionally upper continuous lattice, $C$ be an upper continuous lattice, and $\varphi: L \rightarrow C$ be an upper regular 
lattice-homomorphism. Then there is a unique upper regular latticehomomorphism $\bar{\varphi}: K(L) \rightarrow C$ such that $\bar{\varphi}_{0} \kappa=\varphi$. Moreover, $\bar{\varphi}$ is an injection if and only if $\varphi$ is an injection, and $\bar{\phi}$ is a surjection if and only if $\varphi(L)$ is join-dense in $C$.

Proof. Define $\bar{\varphi}: K(L) \rightarrow C$ by $\bar{\varphi}(A)=\mathrm{V}\{\varphi(x): x \in A\}$ for each $A \in K(L)$.

By 2.1, $a \in \mathrm{V}_{K} A_{\alpha}$ for $\left\{A_{\alpha}\right\} \subseteq K(L)$, implies that $a=\mathrm{V} A$ for some ideal $A \subseteq \bigvee_{J} A_{\alpha}$. For any $x \in A, x \leqq a_{1} \vee a_{2} \vee \cdots \vee a_{n}$ for some $a_{1}, a_{2}, \cdots, a_{n} \in \bigcup A_{\alpha}$. Hence $\varphi(x) \leqq \varphi\left(a_{1}\right) \vee \cdots \vee \varphi\left(a_{n}\right) \leqq \bigvee \bar{\varphi}\left(A_{\alpha}\right)$ and so $\varphi(\alpha)=\bigvee \varphi(A) \leqq \bigvee \bar{\varphi}\left(A_{\alpha}\right)$. Thus $\bar{\varphi}\left(\mathrm{V}_{K} A_{\alpha}\right) \leqq \mathrm{V} \bar{\varphi}\left(A_{\alpha}\right)$ and it follows that $\bar{\phi}$ is upper regular.

Since $C$ is upper continuous $\bar{\varphi}(A) \wedge \bar{\varphi}(B)=\bigvee\{\varphi(a): a \in A\} \wedge$ $\mathrm{V}\{\varphi(b): b \in B\}=\mathrm{V}\{\varphi(a) \wedge \varphi(b): a \in A, b \in B\}=\mathrm{V}\{\varphi(a \wedge b): a \in A, b \in B\}=$ $\bar{\varphi}(A \cap B)$ for any $A, B \in K(L)$.

Clearly $\bar{\varphi}_{0} \kappa=\varphi$. Now suppose $\psi: K(L) \rightarrow C$ is another map possessing the same properties as we have already established for $\bar{\phi}$. Let $A \in K(L)$. Of course $A=\bigcup\{(x]: x \in A\}$ and so $A=\mathrm{V}_{K}\{(x]: x \in A\}=$ $\bigvee_{K}\{\kappa(x): x \in A\}$. Hence $\psi(A)=\bigvee\{\psi(\kappa(x)): x \in A\}=\bigvee\{\varphi(x): x \in A\}=$ $\bar{\varphi}(J)$ and so $\bar{\varphi}$ is unique.

Suppose $\varphi$ is an injection. Let $A, B \in K(L)$ be such that $\bar{\varphi}(A)=$ $\bar{\varphi}(B)$. For $x \in A, \varphi(x)=\bar{\varphi}(\kappa(x))=\bar{\varphi}((x])=\bar{\varphi}((x]) \cap \bar{\varphi}(A)=\bar{\varphi}((x] \cap B)$. If $z \in L$ is an upper bound of $(x] \cap B$ then $\varphi(z)=\bar{\varphi}((z]) \geqq \bar{\varphi}((x] \cap B)=$ $\varphi(x)$. Hence $z \geqq x$ and it follows that $x=\mathrm{V}(x] \cap B \in B$. Thus $A \subseteq$ $B$. Due to symmetry $A=B$ and so $\bar{\phi}$ is an injection. The converse is trivial.

Suppose $\varphi(L)$ is join-dense in $C$. Let $c \in C$. As $\varphi$ is upper regular $\varphi^{-}((c])=\{x \in L: \varphi(x) \leqq c\}$ is a complete ideal. Since $\varphi(L)$ is joindense in $C \bar{\varphi}\left(\varphi^{+}((c])\right)=\mathrm{V}\{\varphi(x): \varphi(x) \leqq c\}=c$. Hence $\bar{\varphi}$ is a surjection. The converse is readily established.

THEOREM 3.2. Let $L$ be a conditionally upper continuous lattice, $P$ be a partially ordered set and $\psi: L \rightarrow P$ be an embedding (i.e., $x \leqq y \Leftrightarrow \psi(x) \leqq \psi(y)$ for any $x, y \in L)$ which is upper regular and such that $\psi(L)$ is join-dense in $P$. Then there exists a unique embedding $\psi^{\prime}: P \rightarrow K(L)$ such that $\psi^{\prime} \circ \psi=\kappa$. In addition $\psi^{\prime}(S)$ is join-dense in $K(L)$ and $\psi^{\prime}$ is a surjection if and only if $P$ is an upper continuous lattice.

Proof. Define $\psi^{\prime}: P \rightarrow K(L)$ by $\psi^{\prime}(p)=\{x \in L: \psi(x) \leqq p\}$ for each $p \in P$. Since $\psi$ is upper regular $\psi^{\prime}(p)$ is always a complete ideal. For $p, q \in P$ it is clear that $\psi^{\prime}(p) \leqq \psi^{\prime}(q)$ if $p \leqq q$ and the converse holds since $\psi(L)$ is join-dense in $P$.

For $x \in L \quad \psi^{\prime}(\psi(x))=\{y \in L: \psi(y) \leqq \psi(x)\}=\{y \in L: y \leqq x\}=\kappa(x)$. 
Now suppose that $\lambda: P \rightarrow K(L)$ is another embedding such that $\lambda \circ \psi=$ $\kappa$. Let $p \in P$ and suppose $x \in L$ and $x \in \psi^{\prime}(p)$. Then $\psi(x) \leqq p$ and so $x \in \kappa(x)=\lambda(\psi(x)) \subseteq \lambda(p)$. That is $\psi^{\prime}(p) \subseteq \lambda(p)$. Now let $p \in P$ and $x \in L$ be such that $x \in \lambda(p)$. Hence $(x] \leqq \lambda(p)$ i.e., $\lambda(\psi(x)) \leqq \lambda(p)$. As $\lambda$ is an embedding, $\psi(x) \leqq p$. Hence $\lambda(p) \subseteq \psi^{\prime}(p)$ and it follows that $\lambda=\psi^{\prime}$.

Clearly $\psi^{\prime}(S)$ is join-dense in $K(L)$ and hence the last assertion of the theorem follows from 3.1 if we note that a join-dense embedding is always lower regular.

CoROllary 3.2.1. For a lattice $L$ the following are equivalent.

(a) The Dedekind-MacNeille completion of $L$ is upper continuous.

(b) $L$ is conditionally upper continuous and $K(L)$ is isomorphic to the Dedekind-MacNeille completion of $L$.

(c) $L$ is upper continuous and $\kappa(L)$ is meet-dense in $K(L)$.

Proof. Up to isomorphism the Dedekind-MacNeille completion of $L$ is the unique join-dense and meet-dense complete extension of $L$ [12].

Dilworth and McLaughlin [3, Theorem 3.2 and Corollary to Theorem 3.4] have already given necessary and sufficient conditions for the Dedekind-MacNeille completion of a lattice to be infinitely distributive. Corollaries 2.2.2 and 3.2.1 yield new conditions.

COROLlaRY 3.2.2. For a lattice $L$ the following are equivalent.

(a) $L$ is distributive and its Dedekind-MacNeille completion is upper continuous.

(b) The Dedekind-MacNeille completion of $L$ is infinitely distributive.

(c) $L$ is infinitely distributive and $K(L)$ is isomorphic to the Dedekind-MacNeille completion of $L$.

(d) $L$ is infinitely distributive and $\kappa(L)$ is meet-dense in $K(L)$.

COROLLARY 3.2.3. The Crawley completion of an implicative lattice is isomorphic to the Dedekind-MacNeille completion.

Proof. Smith [14, Theorem 4.1] has shown that the DedekindMacNeille completion of an implicative lattice is itself an implicative lattice. The result is now an immediate consequence of Corollaries 2.2.2 and 3.2.2.

4. Disjunctive lattices. Let $L$ be a distributive lattice with 0 . For a nonempty subset $A$ of $L$ put $A^{*}=\{x \in L: x \wedge a=0$ for all 
$a \in A\}$ and $A^{* *}=\left(A^{*}\right)^{*}$. Then $A^{*}$ is an ideal and an ideal $B$ is called an annihilator ideal if and only if $B=B^{* *}$ or equivalently if $B=A^{*}$ for some nonempty subset $A$. When the set $A(L)$ of all annihilator ideals of $L$ is ordered by set-inclusion it becomes a complete Boolean algebra and the function $\alpha: L \rightarrow A(L), \alpha(x)=x^{* *}$ for each $x \in L$, is a lattice homomorphism of $L$ into $A(L)$. For details see [6, Lemma 12 , p. 107] or [11, §3].

Recall that a distributive lattice $L$ with 0 is disjunctive if for any $a<b, a, b \in L$, there exists $0<c \in L$ such that $a \wedge c=0$ and $c<b$; "section semi-complemented" is an equivalent term used in [9]. It is well-known that a distributive lattice with 0 is disjunctive if and only if $(x]=(x]^{* *}$ for each $x \in L$, or equivalently the map $\alpha: L \rightarrow$ $A(L)$ is an injection. A subset $S$ is join-dense in a disjunctive lattice $L \neq\{0\}$ if and only if it is dense in the sense of $[11, \S 3]$, that is, for any $0 \neq s \in S$ such that $s \leqq x$. It is also easy to see that a disjunctive lattice $L$ is infinitely distributive if and only if the embedding $\alpha: L \rightarrow A(L)$ is upper regular. Finally we remark that $\alpha(L)$ is join-dense in $A(L)$ for any distributive lattice $L$ with 0 and that $L$ is disjunctive if and only if it is a dense (join-dense) sublattice of a Boolean algebra. Up to isomorphism $A(L)$ is the unique complete Boolean algebra containing disjunctive lattice $L$ as a dense sublattice $[11, \S 3]$.

The preceding remarks together with Corollary 2.2.2 and Theorem 3.2 yield

THEOREM 4.1. For a lattice $L$ with $0, K(L)$ is a complete Boolean algebra if and only if $L$ is disjunctive and infinitely distributive. Under these equivalent conditions $K(L)$ is isomorphic to $A(L)$, the complete Boolean algebra of annihilator ideals.

A dual disjunctive lattice $L$ is a distributive lattice with 1 whose dual is disjunctive. From the preceding remarks it follows that a lattice with 1 is dual disjunctive if and only if it is a meet-dense sublattice of a complete Boolean algebra. Because of [9, Lemma 3.7] a dual disjunctive lattice is infinitely distributive; actually Lemma 3.7 of [9] states that a dual disjunctive lattice is conditionally upper continuous and it may be worth noting that the proof of that lemma implies infinite distributivity. The preceding remarks of this section together with Corollary 3.2.2 yield

THeOREm 4.2. Let $L$ be a lattice with 0 and 1 . The DedekindMacNeille completion of $L$ is a complete Boolean algebra if and only if $L$ is a disjunctive, dual disjunctive distributive lattice. Under these equivalent conditions the Dedekind-MacNeille completion, Crawley 
completion and Boolean algebra of annihilator ideals of $L$ are all isomorphic.

Most of Theorem 4.2 has already been established by Janowitz [9, Lemma 3.9, Theorem 3.11]. Our approach is different and also identifies the Dedekind-MacNeille completion.

Because of Theorem 4.2 it would seem worthwhile exhibiting a class of nonBoolean disjunctive, dual disjunctive lattices. Using some results of Pierce [10], together with an assumed knowledge of the topological notions of [5], we obtain a satisfying class of examples.

THEOREM 4.3. Let $X$ be a completely regular topological space. Let $\mathscr{D}(X)=\{Y: Y \subseteq X, Y=\operatorname{int}(\mathrm{cl} P)$ for some co-zero set in $X\}$ be ordered by set-inclusion. $\mathscr{D}(X)$ is a disjunctive, dual disjunctive lattice where the meet in $\mathscr{D}(X)$ is set-theoretic intersection and the join is given by $A \vee B=\operatorname{int} \operatorname{cl}(A \cup B)$ for any $A, B \in \mathscr{D}(X)$; the Boolean algebra of regular open set of $X$ is the Dedekind-MacNeille completion of $\mathscr{D}(X)$.

$\mathscr{D}(X)$ is a Boolean algebra if and only if for any co-zero set $P$ there exists a co-zero set $Q$ such that $P \cap Q=\varnothing$ and $\operatorname{cl}(P \cup Q)=X$. Hence if $X$ is an $F$-space then $\mathscr{D}(X)$ is Boolean if and only if $X$ is basically disconnected.

Proof. The assertion of the first paragraph of the theorem is a version of [10, Lemmas 2.3, 2.4].

The criterion for $\mathscr{D}(X)$ to be Boolean is an immediate consequence of the description of the join in $\mathscr{D}(X)$. In an $F$-space disjoint co-zero sets have disjoint closures and so it follows that $\mathscr{D}(X)$ is Boolean for an $F$-space $X$ if and only if the closure of each co-zero set is open and closed. The last condition is the defining condition for basically disconnected spaces.

The criterion for $\mathscr{D}(X)$ to be Boolean arises in another context. It is the necessary and sufficient condition for the space of minimal prime ideals of the ring of continuous real-valued functions on $X$ to be compact with respect to the hull-kernel topology. For details see [7, Corollary 5.5, Theorem 5.6]. There are $F$-spaces which are not basically disconnected e.g. $\beta N \backslash N$ and $\beta R \backslash R$ [5].

In closing we would like to mention that we have been unable to find an example of an infinitely distributive disjunctive lattice with 0 and 1 which is not dual disjunctive.

\section{REFERENCES}

1. Garrett Birkhoff, Lattice Theory, 3rd ed., Amer. Math. Soc. Colloq. Publ. 25, 
Amer. Math. Soc., Providence, R.I., 1967.

2. Peter Crawley, Regular embeddings which preserve lattice structure, Proc. Amer. Math. Soc., 13 (1962), 748-752.

3. R. P. Dilworth and J. E. McLaughlin, Distributivity in lattices, Duke Math. J., 19 (1952), 683-694.

4. Nenosuke Funayama, Imbedding infinitely distributive lattices completely isomorphically into Boolean algebras, Nagoya Math. J., 15 (1959), 71-81.

5. Leonard Gillman and Meyer Jerison, Rings of Continuous Functions, Van Nostrand, Princeton, N.J., 1960.

6. George Grätzer, Lattice Theory. First Concepts and Distributive Lattices, W. H. Freeman, San Francisco, 1971.

7. M. Henriksen and M. Jerison, The space of minimal prime ideals of a commutative ring, Trans. Amer. Math. Soc., 115 (1965), 110-130.

8. M. F. Janowitz, On conditionally upper continuous lattices, J. Sci. Hiroshima Univ. Ser. A-1, 32 (1968), 1-4.

9. _- Section semicomplemented lattices, Math. Z., 108 (1968), 63-76.

10. R. S. Pierce, The Boolean algebra of regular open sets, Canad. J. Math., 5 (1953), 95-100.

11. - Translation lattices, Memoirs Amer. Math. Soc., 32 (1959).

12. Jürgen Schmidt, Zur Kennzeichnung der Dedekind-MacNeilleschen Hülle einer geordneten Hülle, Arch. Math. (Basel), 7 (1956), 241-249.

13. —- Zu einem Axiom von Baer in Verbänden, Arch. Math. (Basel), 10 (1959), 104-108.

14. Dorothy P. Smith, Meet-irreducible elements in implicative lattices, Proc. Amer. Math. Soc., 34 (1972), 57-62.

Received February 13, 1973.

The Flinders University of South Australia 



\section{PACIFIC JOURNAL OF MATHEMATICS}

\section{EDITORS}

RICHARD ARENS (Managing Editor)

University of California

Los Angeles, California 90024
J. DUGUNDJI*

Department of Mathematics

University of Southern California

Los Angeles, California 90007

D. Gilbarg and J. Milgram

Stanford University

Stanford, California 94305
University of Washington

Seattle, Washington 98105

ASSOCIATE EDITORS
E. F. BECKENBACH
B. H. NeumanN
F. WoLF
K. YosHIDA

\section{SUPPORTING INSTITUTIONS}

\author{
UNIVERSITY OF BRITISH COLUMBIA \\ CALIFORNIA INSTITUTE OF TECHNOLOGY \\ UNIVERSITY OF CALIFORNIA \\ MONTANA STATE UNIVERSITY \\ UNIVERSITY OF NEVADA \\ NEW MEXICO STATE UNIVERSITY \\ OREGON STATE UNIVERSITY \\ UNIVERSITY OF OREGON \\ OSAKA UNIVERSITY
}

\author{
UNIVERSITY OF SOUTHERN CALIFORNIA \\ STANFORD UNIVERSITY \\ UNIVERSITY OF TOKYO \\ UNIVERSITY OF UTAH \\ WASHINGTON STATE UNIVERSITY \\ UNIVERSITY OF WASHINGTON
* * * *
AMERICAN MATHEMATICAL SOCIETY \\ NAVAL WEAPONS CENTER
}

The Supporting Institutions listed above contribute to the cost of publication of this Journal, but they are not owners or publishers and have no responsibility for its content or policies.

Mathematical papers intended for publication in the Pacific Journal of Mathematics should be in typed form or offset-reproduced, (not dittoed), double spaced with large margins. Underline Greek letters in red, German in green, and script in blue. The first paragraph or two must be capable of being used separately as a synopsis of the entire paper. Items of the bibliography should not be cited there unless absolutely necessary, in which case they must be identified by author and Journal, rather than by item number. Manuscripts, in duplicate if possible, may be sent to any one of the four editors. Please classify according to the scheme of Math. Rev. Index to Vol. 39. All other communications to the editors should be addressed to the managing editor, or Elaine Barth, University of California, Los Angeles, California, 90024.

100 reprints are provided free for each article, only if page charges have been substantially paid. Additional copies may be obtained at cost in multiples of 50 .

The Pacific of Journal Mathematics is issued monthly as of January 1966. Regular subscription rate: $\$ 72.00$ a year (6 Vols., 12 issues). Special rate: $\$ 36.00$ a year to individual members of supporting institutions.

Subscriptions, orders for back numbers, and changes of address should be sent to Pacific Journal of Mathematics, 103 Highland Boulevard, Berkeley, California, 94708.

PUBLISHED BY PACIFIC JOURNAL OF MATHEMATICS, A NON-PROFIT CORPORATION

Printed at Kokusai Bunken Insatsusha (International Academic Printing Co., Ltd.), 270, 3-chome Totsuka-cho, Shinjuku-ku, Tokyo 160, Japan.

* C. R. DePrima California Institute of Technology, Pasadena, CA 91109, will replace J. Dugundji until August 1974.

Copyright (C) 1973 by Pacific Journal of Mathematics

Manufactured and first issued in Japan 


\section{Pacific Journal of Mathematics}

\section{Vol. 51, No. $2 \quad$ December, 1974}

Robert F. V. Anderson, Laplace transform methods in multivariate spectral theory .................................................. 339

William George Bade, Two properties of the Sorgenfrey plane . . . . . . . . . . . . 349

John Robert Baxter and Rafael Van Severen Chacon, Functionals on continuous

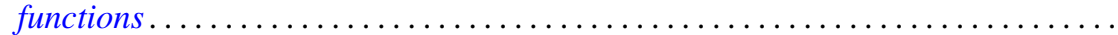

Phillip Wayne Bean, Helly and Radon-type theorems in interval convexity

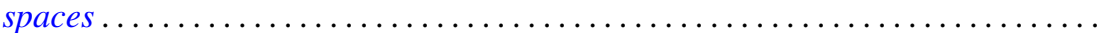

James Robert Boone, On k-quotient mappings $\ldots \ldots \ldots \ldots \ldots \ldots \ldots \ldots \ldots$

Ronald P. Brown, Extended prime spots and quadratic forms . . . . . . . . . . . .

William Hugh Cornish, Crawley's completion of a conditionally upper continuous lattice .............................................

Robert S. Cunningham, On finite left localizations ...................

Robert Jay Daverman, Approximating polyhedra in codimension one spheres

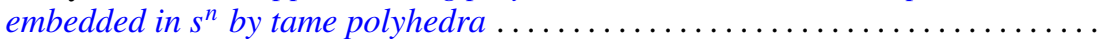

Burton I. Fein, Minimal splitting fields for group representations . . . . . . . . . . . .

Peter Fletcher and Robert Allen McCoy, Conditions under which a connected

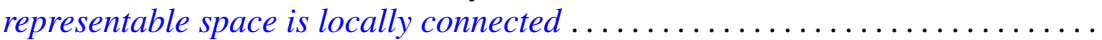

Jonathan Samuel Golan, Topologies on the torsion-theoretic spectrum of a noncommutative ring...

Manfred Gordon and Edward Martin Wilkinson, Determinants of Petrie matrices.

Alfred Peter Hallstrom, A counterexample to a conjecture on an integral condition for determining peak points (counterexample concerning peak points)........

E. R. Heal and Michael Windham, Finitely generated $F$-algebras with applications to Stein manifolds.

Denton Elwood Hewgill, On the eigenvalues of a second order elliptic operator in an unbounded domain ............................

Charles Royal Johnson, The Hadamard product of $A$ and $A^{*}$.

Darrell Conley Kent and Gary Douglas Richardson, Regular completions of Cauchy spaces.

Alan Greenwell Law and Ann L. McKerracher, Sharpened polynomial approximation

Bruce Stephen Lund, Subalgebras of finite codimension in the algebra of analytic functions on a Riemann surface. .

Robert Wilmer Miller, TTF classes and quasi-generators . .

Roberta Mura and Akbar H. Rhemtulla, Solvable groups in which every maximal partial order is isolated ....

Isaac Namioka, Separate continuity and joint continuity...

Alan Saleski, Entropy of self-homeomorphisms of statistical pseudo-metric

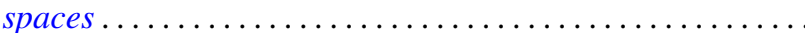

H. A. Seid, Cyclic multiplication operators on $L_{p}$-spaces .....

H. B. Skerry, On matrix maps of entire sequences ............

John Brendan Sullivan, A proof of the finite generation of invariants of a normal

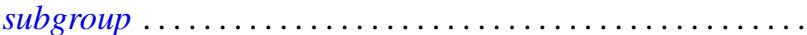

John Griggs Thompson, Nonsolvable finite groups all of whose local subgroups are

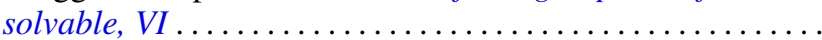

